\title{
ON A WEIGHTED TOEPLITZ OPERATOR AND ITS COMMUTANT
}

\author{
VASILE LAURIC
}

Received 14 July 2004 and in revised form 28 February 2005

We study the structure of a class of weighted Toeplitz operators and obtain a description of the commutant of each operator in this class. We make some progress towards proving that the only operator in the commutant which is not a scalar multiple of the identity operator and which commutes with a nonzero compact operator is zero. The proof of the main statement relies on a conjecture which is left as an open problem.

\section{Introduction}

Let $\mathscr{H}$ be a separable, infinite dimensional, complex Hilbert space and denote by $\mathscr{L}(\mathscr{H})$ the algebra of all bounded linear operators on $\mathscr{H}$. We introduce some notation to easily state the main consequence of this paper. Define

$$
\begin{gathered}
\mathscr{S}:=\left\{T \in \mathscr{L}(\mathscr{H}) \mid \exists A \in \mathscr{L}^{\prime}(\mathscr{H}), \exists K \in \mathbb{K} \backslash\{0\},[T A=A T \wedge A K=K A]\right\}, \\
\tilde{\mathscr{S}}:=\left\{T \in \mathscr{L}(\mathscr{H}) \mid \exists A \in \mathscr{L}^{\prime}(\mathscr{H}), \exists K \in \mathbb{K} \backslash\{0\}, \exists \alpha \in \mathbb{C},[T A=A T \wedge A K=\alpha K A]\right\},
\end{gathered}
$$

where $\mathscr{L}^{\prime}(\mathscr{H})$ is the set of all operators in $\mathscr{L}(\mathscr{H})$ that are not scalar multiples of the identity operator and $\mathbb{K}$ is the ideal of compact operators in $\mathscr{L}(\mathscr{H})$. The sets $\mathscr{Y}$ and $\tilde{\mathscr{S}}$ are suggested by Lomonosov's celebrated result concerning the existence of hyperinvariant subspaces, as well as, some generalization obtained by Brown [2] and Kim et al. [7].

Recently, some progress was made by Biswas et al. [1], on one hand, in describing the set of values that $\alpha$ can assume, as well as, the associated operators $X$ that satisfy the equality $X A=\alpha A X$ for some given operator $A$, and by Conway and Prăjitură [4], on the other hand, concerning the structure of such operators.

In [6], it was proved that some weighted shift operator does not meet the hypothesis of Lomonosov's theorem, that is, it does not belong to the set $\mathscr{Y}$. Although it is expected that the set $\tilde{\mathscr{S}}$ be larger than the set $\mathscr{Y}$, it is not clear whether the difference between these two sets is significant. In Section 3 of this paper, we make some progress towards proving that there are indeed many operators in the $\widetilde{\mathscr{S}} \backslash \mathscr{Y}$, and therefore it is worth while to study the question of whether $\tilde{\mathscr{S}}$ is the entire $\mathscr{L}(\mathscr{H})$. 
The class of operators that we will investigate consists of some weighted Toeplitz operators. In [9], Shields made a comprehensive study of the class of weighted shift operators. In order to define our class of weighted Toeplitz operators, we recall some notation from [9]. Although the definition can be given in a more general setting, we prefer to impose some restrictions.

Let $\beta=\left(\beta_{n}\right)_{n \in \mathbb{Z}}$ be a sequence of positive numbers with $\beta_{0}=1$ and such that

$$
\sum_{n \in \mathbb{Z}} \frac{1}{\beta_{n}^{2}}<\infty, \quad m \leq \frac{\beta_{n}}{\beta_{n+1}} \leq M \quad \text { where } 0<m \leq M<\infty, n \in \mathbb{N} .
$$

The space $L^{2}(\beta)$ is

$$
\left\{f(z)=\left.\sum_{n=-\infty}^{\infty} a_{n} z^{n}\left|a_{n} \in \mathbb{C},\|f\|_{\beta}^{2}:=\sum_{n=-\infty}^{\infty}\right| a_{n}\right|^{2} \beta_{n}^{2}<\infty\right\},
$$

and let $H^{2}(\beta)$ be the subspace of $L^{2}(\beta)$ consisting of

$$
\left\{f(z)=\left.\sum_{n=0}^{\infty} a_{n} z^{n}\left|a_{n} \in \mathbb{C},\|f\|_{\beta}^{2}:=\sum_{n=0}^{\infty}\right| a_{n}\right|^{2} \beta_{n}^{2}<\infty\right\} .
$$

Thus $\left(L^{2}(\beta),\|\cdot\|_{\beta}\right)$ is a Hilbert space with an orthonormal basis $\left\{e_{n}(z)=z^{n} / \beta_{n}\right\}_{n \in \mathbb{Z}}$ (with respect to the appropriate scalar product), and $\left(H^{2}(\beta),\|\cdot\|_{\beta}\right)$ is a subspace of $L^{2}(\beta)$. Let $P: L^{2}(\beta) \rightarrow H^{2}(\beta)$ be the orthogonal projection of $L^{2}(\beta)$ onto $H^{2}(\beta)$. By $L^{\infty}(\beta)$ one denotes the set

$$
\left\{\phi(z)=\sum_{n=-\infty}^{\infty} a_{n} z^{n} \mid \phi L^{2}(\beta) \subseteq L^{2}(\beta) \text { and } \exists c \in \mathbb{R} \text { such that }\|\phi f\|_{\beta} \leq c\|f\|_{\beta}, \forall f \in L^{2}(\beta)\right\} \text {, }
$$

and by $\|\phi\|_{\infty}$ the norm inf $\left\{c \mid\|\phi f\|_{\beta} \leq c\|f\|_{\beta}\right.$, for all $\left.f \in L^{2}(\beta)\right\}$.

A weighted Toeplitz operator on $H^{2}(\beta)$ of symbol $\phi \in L^{\infty}(\beta)$ is defined by $T_{\phi} f:=$ $P(\phi f)$. In this paper, we study the particular class of weighted Toeplitz operators with symbol in the set

$$
\mathscr{G}:=\left\{\phi\left|\phi(z)=a z^{k}+\frac{b}{z^{k}}, k \in \mathbb{N},\right| a|>| b \mid\right\}
$$

and prove that such operators are unitarily equivalent to multiplication by the position operator (to be defined later). As an application of this, we describe the commutant of such a weighted Toeplitz operator.

For purposes of determining the commutant of weighted Toeplitz operators of symbol $\phi \in \mathscr{G}$, we can assume that each symbol is of the form $\phi(z)=\phi_{b}(z):=z+(b / z)$ with $|b|<1$. An easy calculation shows that $\phi_{b}$ belongs to $L^{\infty}(\beta)$ and $\left\|\phi_{b}\right\|_{\infty} \leq 1 / m+|b| M$. Since for $b=0$ one gets the weighted shift operator, we will assume that $0<|b|<1$. 
The matrix representation of such a weighted Toeplitz operator with respect to the orthonormal basis $\left\{e_{n}\right\}_{n=0}^{\infty}$ of $H^{2}(\beta)$ is

$$
T=T_{\phi}^{(\beta)}:=\left(\begin{array}{cccccc}
0 & b \frac{\beta_{0}}{\beta_{1}} & 0 & \ldots & 0 & \cdots \\
\frac{\beta_{1}}{\beta_{0}} & 0 & b \frac{\beta_{1}}{\beta_{2}} & \ldots & 0 & \ldots \\
0 & \frac{\beta_{2}}{\beta_{1}} & 0 & \ddots & 0 & \ldots \\
\vdots & \vdots & \ddots & \ddots & b \frac{\beta_{n-1}}{\beta_{n}} & \ldots \\
0 & 0 & 0 & \frac{\beta_{n}}{\beta_{n-1}} & 0 & \ddots \\
\vdots & \vdots & \vdots & \vdots & \ddots & \ddots
\end{array}\right) .
$$

In what follows, we consider a weighted Toeplitz operator of symbol $\phi_{b}(z)=z+(b / z)$ to be an operator on $l^{2}(\mathbb{N})$, (the Hilbert space of the square summable sequences) whose matrix representation with respect to the canonical basis of $l^{2}(\mathbb{N})$ is of the form (1.7). Next we review some facts about the operator defined above. We begin by describing the point spectrum of the adjoint operator of $T, \sigma_{p}\left(T^{*}\right)$. A complex number $\bar{\lambda}$ belongs to $\sigma_{p}\left(T^{*}\right)$ if and only if there is a nonzero square summable sequence $f=\left\{f_{0}, f_{1}, \ldots, f_{n}, \ldots\right\}$ that satisfies the recurrence equations

$$
\bar{b} \frac{\beta_{n-1}}{\beta_{n}} f_{n-1}-\bar{\lambda} f_{n}+\frac{\beta_{n+1}}{\beta_{n}} f_{n+1}=0, \quad n \in \mathbb{N},
$$

with $f_{-1}:=0$. If one denotes $f_{n} \beta_{n}$ by $p_{n}$, for each $n \in \mathbb{N}$, then the above recurrence equations become

$$
\bar{b} p_{n-1}-\bar{\lambda} p_{n}+p_{n+1}=0, \quad n \in \mathbb{N}
$$

with $p_{-1}:=0$. One can easily see by induction after setting $p_{0}:=1$ that the formal solution of (1.9) is of the form $p_{n}=p_{n}(\bar{\lambda}), n \geq 1$, where $p_{n}(\cdot)$ is a polynomial of degree $n$. If the characteristic equation of recurrence (1.9), $\bar{b}-\bar{\lambda} z+z^{2}=0$, has different roots $z_{i}=z_{i}(\bar{\lambda})$, $i=1,2$, then $p_{n}(\bar{\lambda})$ has the form

$$
p_{n}(\bar{\lambda})=A z_{1}^{n}+B z_{2}^{n}, \quad n \in \mathbb{N},
$$

where $A, B$ do not depend on $n$, but only on $\bar{\lambda}$. On the other hand, if the characteristic equation has a double $\operatorname{root} z_{0}=z_{0}(\bar{\lambda})=\bar{\lambda} / 2= \pm \sqrt{\bar{b}}$, where $\sqrt{\bar{b}}$ is the main complex root of $\bar{b}$, then

$$
p_{n}(\bar{\lambda})=(n+1) z_{0}^{n}, \quad n \in \mathbb{N} .
$$

The characteristic equation $\bar{b}-\bar{\lambda} z+z^{2}=0$ is equivalent to $\phi_{\bar{b}}(z)=\bar{\lambda}$, where $\phi_{\bar{b}}(z)=z+$ $(\bar{b} / z)$. If one denotes by $\mathscr{C}_{\rho}$ the circle $\{z|| z \mid=\rho\}$ and by $\mathscr{E}_{\rho}$ the image of $\mathscr{C}_{\rho}$ under $\phi_{\bar{b}}$, that is, $\phi_{\bar{b}}\left(\mathscr{C}_{\rho}\right)$, then one can see that $\phi_{\bar{b}}$ maps $\mathscr{C}_{\rho}$ and $\mathscr{C}_{r / \rho}$, where $r:=|b|$, onto the same 
ellipse, $\mathscr{E}_{\rho}=\mathscr{E}_{r} / \rho$. In particular, $\mathscr{C}_{1}=\mathbb{T}$ and $\mathscr{C}_{r}$ are mapped onto the same ellipse, denoted by $\mathscr{E}_{T}$. Moreover, the bounded component of $\mathbb{C} \backslash \mathscr{E}_{T}$, denoted by $\operatorname{Int}\left(\mathscr{E}_{T}\right)$, is swept exactly twice as the value of $\rho$ varies from $|b|$ to 1 .

In what follows, we will impose some restrictions on the sequence $\left\{\beta_{n}\right\}_{n}$. The first such restriction is that $\left\{\beta_{n}\right\}_{n}$ is such that the series $\sum_{n=0}^{\infty}\left(z^{2 n} / \beta_{n}^{2}\right)$ is absolutely convergent if and only if $|z| \leq 1$. An example of such a sequence is $\beta_{n}=\beta^{\sqrt{n}}$, with $\beta>1$.

If $\bar{\lambda}= \pm 2 \sqrt{\bar{b}}$, then $\left|z_{0}\right|<1$ according to the earlier assumption that $|b|<1$, and according to (1.11), the sequence $\left\{f_{n}(\bar{\lambda})\right\}_{n \in \mathbb{N}}$ is square summable. On the other hand, if $\bar{\lambda}$ is the image through $\phi_{\bar{b}}(\cdot)$ of two different $z^{\prime}$ s, then taking into consideration equality (1.10), the fact that $f_{n}(\bar{\lambda})=p_{n}(\bar{\lambda}) / \beta_{n}$, and the above assumption about the sequence $\left\{\beta_{n}\right\}_{n}$, the sequence $\left\{f_{n}(\bar{\lambda})\right\}_{n \in \mathbb{N}}$ is square summable if and only if $\left|z_{1}\right| \leq 1$ and $\left|z_{2}\right| \leq 1$. Therefore, the point spectrum of $T^{*}$ includes the set $\operatorname{Int}\left(\mathscr{E}_{T}\right) \cup \mathscr{E}_{T}$ and $\operatorname{dim} \operatorname{Ker}\left(T^{*}-\bar{\lambda}\right)=1$, for $\bar{\lambda} \in \operatorname{Int}\left(\mathscr{E}_{T}\right) \cup \mathscr{E}_{T}$. Using similar arguments, one can see that $\operatorname{dim} \operatorname{Ker}(T-\lambda)=0$, for any $\lambda \in \mathbb{C}$ and that any $\bar{\lambda}$ off the set $\operatorname{Int}\left(\mathscr{E}_{T}\right) \cup \mathscr{E}_{T}$ is not in $\sigma_{p}\left(T^{*}\right)$. For each $\bar{\lambda} \in \operatorname{Int}\left(\mathscr{E}_{T}\right) \cup \mathscr{E}_{T}$, we denote by $f_{\bar{\lambda}}$ the unique vector $\left\{f_{n}(\bar{\lambda})\right\}_{n \in \mathbb{N}}$ in $l^{2}(\mathbb{N})$ that satisfies the recurrence equations (1.8) above with $f_{0}(\bar{\lambda})=1$, that is, $T^{*} f_{\bar{\lambda}}=\bar{\lambda} f_{\bar{\lambda}}$.

Moreover, the series $\sum_{n=0}^{\infty}\left|p_{n}(\bar{\lambda})\right|$ (thus $\left.\sum_{n=0}^{\infty}\left|p_{n}(\bar{\lambda})\right|^{2}\right)$ converges uniformly for $\bar{\lambda}$ in any compact subset of $\operatorname{Int}\left(\mathscr{E}_{T}\right)$ and the series $\sum_{n=0}^{\infty}\left|f_{n}(\bar{\lambda})\right|^{2}$ converges uniformly for $\bar{\lambda} \in$ $\operatorname{Int}\left(\mathscr{E}_{T}\right) \cup \mathscr{E}_{T}$.

Next, we will show that the operator $T$ is unitarily equivalent to multiplication by position operator, $M_{\lambda}$, defined on some Hilbert space of functions. We denote by $\bar{f}_{n}(\lambda)$ the polynomial $\overline{f_{n}(\bar{\lambda})}$ of degree $n$ in variable $\lambda$. We define

$$
H^{2}(G):=\left\{k(\lambda)=\left.\sum_{n=0}^{\infty} h_{n} \bar{f}_{n}(\lambda)\left|h_{n} \in \mathbb{C},\|k\|_{H^{2}}^{2} \stackrel{\text { def }}{=} \sum_{n=0}^{\infty}\right| h_{n}\right|^{2}<\infty\right\} .
$$

According to the above observation concerning the uniform convergence of the series $\sum_{n=0}^{\infty}\left|\bar{f}_{n}(\lambda)\right|^{2}$ for $\lambda \in G:=\left(\operatorname{Int}\left(\mathscr{E}_{T}\right) \cup \mathscr{E}_{T}\right)^{*}$, each element of $H^{2}(G)$ is a continuous function on $G$ and analytic in $\left(\operatorname{Int}\left(\mathscr{E}_{T}\right)\right)^{*}$, where “*” used in conjunction with a subset of $\mathbb{C}$ represents complex conjugation.

\section{The commutant of $T$}

We begin with some notation that is necessary in what follows. Define $U: l^{2}(\mathbb{N}) \rightarrow H^{2}(G)$ by

$$
(U h)(\lambda)=\left\langle h, f_{\bar{\lambda}}\right\rangle_{l^{2}(\mathbb{N})}=\sum_{n=0}^{\infty} h_{n} \bar{f}_{n}(\lambda)
$$

for $h=\left\{h_{n}\right\}_{n=0}^{\infty} \in l^{2}(\mathbb{N})$, and $\lambda \in G$.

We recall that the polynomials $p_{n}(\cdot), n \in \mathbb{N}$, have the following orthogonality property (see, e.g., [5]):

$$
\frac{1}{2 \pi} \int_{\mathscr{E}_{\rho}^{*}} p_{n}(z) \overline{p_{m}(z)} \omega(z)|d z|= \begin{cases}0, & m \neq n, \\ \rho^{2(n+1)}+\left(\frac{r}{\rho}\right)^{2(n+1)}, & m=n\end{cases}
$$


for each $\rho>0$, where $\omega(z)=\left|z^{2}-4 \bar{b}\right|^{1 / 2}, r=|b|$, and $|d z|$ represents arc-length measure. Since $\bar{f}(\lambda)=\bar{p}(\lambda) / \beta_{n}$, we have for $\bar{\lambda} \in \mathscr{E}_{T}$,

$$
\int_{\mathscr{E}_{T}} f_{n}(\bar{\lambda}) \overline{f_{m}(\bar{\lambda})} \omega(\bar{\lambda})|d \bar{\lambda}|= \begin{cases}0, & m \neq n \\ \frac{2 \pi}{\beta_{n}^{2}}\left[1+r^{2(n+1)}\right], & m=n .\end{cases}
$$

The orthogonality property (2.3) implies that $U$ is one-to-one. Obviously, $U$ is linear, and by definition, $\|U h\|_{H^{2}}=\|h\|_{l^{2}(\mathbb{N})}$, and $U$ is an onto isometry. Therefore $U$ is a unitary operator and $H^{2}(G)$ is a Hilbert space. We define

$$
H^{\infty}(G)=\left\{\phi: G \longrightarrow \mathbb{C} \mid \phi H^{2}(G) \subseteq H^{2}(G) \text { such that the inclusion is continuous }\right\},
$$

with $\|\phi\|_{\infty}=\inf \left\{c \mid\|\phi k\|_{H^{2}} \leq c\|k\|_{H^{2}}\right.$, for all $\left.k \in H^{2}(G)\right\}$. Since the function 1 (constant function equal to 1 ) belongs to $H^{2}(G)$ and $\|1\|_{H^{2}}=1$, we have $\|\phi\|_{H^{2}} \leq\|\phi\|_{\infty}$ and the inclusion $H^{\infty}(G) \subseteq H^{2}(G)$ is continuous. (We will see later that equality holds in some cases.) The recurrence equation (1.8) is equivalent to

$$
\lambda \bar{f}_{n}(\lambda)=b \frac{\beta_{n-1}}{\beta_{n}} \bar{f}_{n-1}(\lambda)+\frac{\beta_{n+1}}{\beta_{n}} \bar{f}_{n+1}(\lambda), \quad n \in \mathbb{N} .
$$

If $k \in H^{2}(G)$ is written as $\sum_{n=0}^{\infty} h_{n} \bar{f}_{n}(\lambda)$, then $\lambda k(\lambda)=\sum_{n=0}^{\infty} g_{n} \bar{f}_{n}(\lambda)$, where

$$
g_{n}=\frac{\beta_{n}}{\beta_{n-1}} h_{n-1}+b \frac{\beta_{n}}{\beta_{n+1}} h_{n+1}, \quad n \in \mathbb{N},
$$

with $h_{-1}=0$. Therefore,

$$
\|\lambda k(\lambda)\|_{H^{2}}^{2}=\sum_{n=0}^{\infty}\left|g_{n}\right|^{2} \leq \sum_{n=0}^{\infty}\left(\frac{1}{m}\left|h_{n-1}\right|+M|b|\left|h_{n+1}\right|\right)^{2} \leq\left(\frac{1}{m}+M|b|\right)^{2}\|k\|_{H^{2}}^{2},
$$

which implies that the function $\lambda \mapsto \lambda$ belongs to $H^{\infty}(G)$ and thus, $H^{\infty}(G)$ includes all polynomials of variable $\lambda$. Let $M_{\lambda}$ be the operator defined on $H^{2}(G)$ by $\left(M_{\lambda} k\right)(\lambda)=\lambda k(\lambda)$. The above inequality shows that $M_{\lambda} \in \mathscr{L}\left(H^{2}(G)\right)$ and $\left\|M_{\lambda}\right\| \leq 1 / m+M|b|$. The following proposition asserts the unitary equivalence of $T$ and $M_{\lambda}$.

Proposition 2.1. The operators $U, T$, and $M_{\lambda}$ satisfy the equality $U T=M_{\lambda} U$.

Proof. Let $h$ be an arbitrary vector in $l^{2}(\mathbb{N})$ and let $\lambda$ be in $G$ and observe that

$$
U(T h)(\lambda)=\left\langle T h, f_{\bar{\lambda}}\right\rangle=\left\langle h, T^{*} f_{\bar{\lambda}}\right\rangle=\left\langle h, \bar{\lambda} f_{\bar{\lambda}}\right\rangle=\lambda\left\langle h, f_{\bar{\lambda}}\right\rangle=M_{\lambda}(U h)(\lambda) .
$$

The operator $M_{\lambda}$ admits an $H^{\infty}(G)$-calculus, that is, there exists a continuous homomorphism $\Gamma_{1}: H^{\infty}(G) \rightarrow \mathscr{L}\left(H^{2}(G)\right)$ defined by $\left(\Gamma_{1}(\phi) k\right)(\lambda):=\phi(\lambda) k(\lambda)$. We write $M_{\phi(\lambda)}$ (or simply $M_{\phi}$ ) for $\Gamma_{1}(\phi)$. One can define an $H^{\infty}(G)$-calculus for $T$ as follows:

$$
\Gamma_{2}: H^{\infty}(G) \longrightarrow \mathscr{L}\left(l^{2}(\mathbb{N})\right), \quad \Gamma_{2}(\phi):=U^{*} M_{\phi} U
$$


Obviously, $\Gamma_{2}$ is a continuous homomorphism which extends the polynomial calculus. We write $\phi(T)$ for $\Gamma_{2}(\phi)$ and observe that $T \phi(T)=\phi(T) T$, for any $\phi \in H^{\infty}(G)$.

To describe the commutant of $T,\{T\}^{\prime}$, it is enough to find the commutant of $M_{\lambda}$, $\left\{M_{\lambda}\right\}^{\prime}$. The description of $\left\{M_{\lambda}\right\}^{\prime}$ is similar to that of the commutant of the Bergman (or Hardy) operator, but it requires some details.

Theorem 2.2. $\left\{M_{\lambda}\right\}^{\prime}=\left\{M_{\phi} \mid \phi \in H^{\infty}(G)\right\}$.

Since $A \in\{T\}^{\prime}$ if and only if $U A U^{*} \in\left\{M_{\lambda}\right\}^{\prime}$, we have the following.

Corollary 2.3. $\{T\}^{\prime}=\left\{\phi(T) \mid \phi \in H^{\infty}(G)\right\}$.

Since $f_{\bar{\lambda}} \in l^{2}(\mathbb{N})$, we can set $U f_{\bar{\lambda}}=: k_{\bar{\lambda}}$ for $\lambda \in G$, and thus $k_{\bar{\lambda}}$ belongs to $H^{2}(G)$. Moreover, since the set of all $f_{\bar{\lambda}}$ has the spanning property (an easy consequence of orthogonality property $(2.3)$ ), so does the set of all $k_{\bar{\lambda}}$, that is, the closed linear span of all $k_{\bar{\lambda}}$, $\vee\left\{k_{\bar{\lambda}} \mid \lambda \in G\right\}$, is equal to $H^{2}(G)$.

The following lemma is needed in what follows.

LEMma 2.4. If $k \in H^{2}(G)$ and $\lambda \in G$, then $\left\langle k, k_{\lambda}\right\rangle_{H^{2}(G)}=k(\lambda)$.

Proof. We first observe that

$$
k_{\bar{\lambda}}(w)=\left(U f_{\bar{\lambda}}\right)(w)=\left\langle f_{\bar{\lambda}}, f_{\bar{w}}\right\rangle_{l^{2}(\mathbb{N})}=\sum_{n \in \mathbb{N}} f_{n}(\bar{\lambda}) \bar{f}_{n}(w) \quad \text { for } w \in G
$$

If $k \in H^{2}(G)$ is written as $k(\lambda)=\sum_{n=0}^{\infty} h_{n} \bar{f}_{n}(\lambda)$, then by definition, $\left\langle k, k_{\bar{\lambda}}\right\rangle_{H^{2}(G)}$ is equal to $\sum_{n=0}^{\infty} h_{n} \bar{f}_{n}(\lambda)$, which is equal to $k(\lambda)$.

Proof of Theorem 2.2. Obviously, an operator $A$ in $\mathscr{L}\left(H^{2}(G)\right)$ belongs to $\left\{M_{\lambda}\right\}^{\prime}$ if and only if $A^{*} M_{\lambda}^{*}=M_{\lambda}^{*} A^{*}$. Applying this equality to $k_{\bar{\lambda}}$, we obtain

$$
A^{*} M_{\lambda}^{*} k_{\bar{\lambda}}=M_{\lambda}^{*} A^{*} k_{\bar{\lambda}} \quad \forall \lambda \in G .
$$

Since $U T^{*}=M_{\lambda}^{*} U$, we get that $U T^{*} f_{\bar{\lambda}}=M_{\lambda}^{*} U f_{\bar{\lambda}}$ for $\lambda \in G$, and thus $U \bar{\lambda} f_{\bar{\lambda}}=M_{\lambda}^{*} U f_{\bar{\lambda}}$ for $\lambda \in G$, that is,

$$
\bar{\lambda} k_{\bar{\lambda}}=M_{\lambda}^{*} k_{\bar{\lambda}} \quad \forall \lambda \in G
$$

Combining equalities (2.11) and (2.12), we have that $A^{*} k_{\bar{\lambda}} \in \operatorname{Ker}\left(M_{\lambda}^{*}-\bar{\lambda}\right)$ for $\lambda \in G$. Since $\operatorname{dim} \operatorname{Ker}\left(M_{\lambda}^{*}-\bar{\lambda}\right)=1, A^{*} k_{\bar{\lambda}}=\phi(\bar{\lambda}) k_{\bar{\lambda}}$, where $\phi(\bar{\lambda}) \in \mathbb{C}$ for $\lambda \in G$. We will show that $\overline{\phi(\cdot)} \in H^{\infty}(G)$. Indeed, for an arbitrary $k$ in $H^{2}(G)$,

$$
\overline{\phi(\bar{\lambda})} k(\lambda)=\overline{\phi(\bar{\lambda})}\left\langle k, k_{\bar{\lambda}}\right\rangle_{H^{2}}=\left\langle k, \phi(\bar{\lambda}) k_{\bar{\lambda}}\right\rangle_{H^{2}}=\left\langle k, A^{*} k_{\bar{\lambda}}\right\rangle_{H^{2}}=\left\langle A k, k_{\bar{\lambda}}\right\rangle_{H^{2}}=(A k)(\lambda) .
$$

Since $A k \in H^{2}(G)$ for every $k \in H^{2}(G)$, we have $\bar{\phi}(\lambda):=\overline{\phi(\bar{\lambda})} \in H^{\infty}(G)$. Moreover, the above equalities show that $A=M_{\bar{\phi}}$. Therefore $\left\{M_{\lambda}\right\}^{\prime} \subseteq\left\{M_{\phi} \mid \phi \in H^{\infty}(G)\right\}$. The reverse inclusion is obvious since $\Gamma_{1}$ is an $H^{\infty}(G)$-calculus for $M_{\lambda}$. 


\section{Application and remarks}

In [8], it was proved that every Toeplitz operator or weighted Toeplitz operator, say $T$, with symbol in the set $\mathscr{G}$ has a neighborhood 0 in $\mathbb{C}$ such that for each $\alpha \in \mathbb{O}$ there exists a nonzero compact operator $K$ such that $T K=\alpha K T$. Obviously then, each weighted Toeplitz operator $T$ with symbol in the set $\mathscr{G}$ commutes with itself, and thus with an operator which is not a scalar multiple of the identity operator, which, in turns, $\alpha$-commutes with a nonzero compact operator. Therefore weighted Toeplitz operators with symbol in

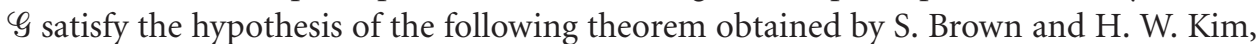
R. Moore, and C. M. Pearcy, that is, such operators belong to $\tilde{\mathscr{T}}$.

Theorem $3.1[2,7]$. If $A \in \mathscr{L}(\mathcal{H})$ is not a scalar multiplication of the identity operator and there exists a nonzero compact operator $K$ such that $A K=\alpha K A$ for some complex number $\alpha$, then $A$ has a nontrivial hyperinvariant subspace.

The purpose of this section is to set up the steps for showing that the weighted Toeplitz operators with symbol in the set $\mathscr{G}$ do not satisfy the hypothesis of Lomonosov's result and to prove some of these steps. Nevertheless, one of these steps cannot be established and is left as an open problem (see Conjecture 3.6 below).

We prepare the ground for applying the main result of [6]. From now on, we will work under the assumption that $\beta_{n}=\beta^{\sqrt{n}}$, although the results might be proved under more general hypothesis.

Lemma 3.2. Let $w \in\left(\operatorname{Int}\left(\mathscr{E}_{T}\right)\right)^{*}$ and $\beta_{n}=\beta^{\sqrt{n}}$ with $\beta>1$. If $\phi(w)=0$ for some $\phi \in H^{\infty}(G)$ (or $H^{2}(G)$ ), then $\phi(\lambda)=(\lambda-w) \psi(\lambda)$ with $\psi \in H^{\infty}(G)$ (or $H^{2}(G)$ ), respectively.

Proof. Let $\phi$ be in $H^{\infty}(G)$ with $\phi(w)=0$. Since $H^{\infty}(G) \subseteq H^{2}(G)$, we can write $\phi(\lambda)$ as

$$
\sum_{n=0}^{\infty} \phi_{n} \bar{f}_{n}(\lambda) \quad \text { or } \quad \sum_{n=0}^{\infty} \frac{\phi_{n}}{\beta_{n}} \bar{p}_{n}(\lambda)
$$

where $\bar{p}_{n}(\lambda):=\overline{p_{n}(\bar{\lambda})}$. Therefore,

$$
\frac{\phi(\lambda)}{\lambda-w}=\sum_{n=0}^{\infty} \frac{\phi_{n}}{\beta_{n}} \frac{\bar{p}_{n}(\lambda)-\bar{p}_{n}(w)}{\lambda-w} .
$$

Using (1.9) and an induction argument, one can deduce that

$$
\frac{\bar{p}_{s}(\lambda)-\bar{p}_{s}(w)}{\lambda-w}=\sum_{r=0}^{s-1} \bar{p}_{r}(w) \cdot \bar{p}_{s-r-1}(\lambda) \quad \forall s \in \mathbb{N} .
$$

Therefore, according to (3.3), we obtain that

$$
\psi(\lambda):=\frac{\phi(\lambda)}{\lambda-w}=\sum_{n=0}^{\infty} \psi_{n} \bar{p}_{n}(\lambda)
$$


where

$$
\psi_{n}=\sum_{s=0}^{\infty} \frac{\phi_{n+s+1}}{\beta_{n+s+1}} \bar{p}_{s}(w) .
$$

We will first prove that $\sum_{n=0}^{\infty}\left|\psi_{n}\right|^{2} \beta_{n}^{2}$ is finite, that is, $\psi \in H^{2}(G)$. Indeed, we have

$$
\left|\psi_{n}\right|^{2} \leq P(w)\left(\sum_{s=0}^{\infty} \frac{\left|\phi_{n+s+1}\right|^{2}}{\beta_{n+s+1}^{2}} \cdot\left|\bar{p}_{s}(w)\right|\right),
$$

where $P(w)=\sum_{n=0}^{\infty}\left|\bar{p}_{s}(w)\right|$, which is finite since $w \in\left(\operatorname{Int}\left(\mathscr{E}_{T}\right)\right)^{*}$. Thus

$$
\begin{aligned}
\|\psi\|_{H^{2}}^{2} & \leq P(w) \sum_{n=0}^{\infty}\left(\sum_{s=0}^{\infty} \frac{\left|\phi_{n+s+1}\right|^{2}}{\beta_{n+s+1}^{2}} \cdot\left|\bar{p}_{s}(w)\right|\right) \beta_{n}^{2} \\
& =P(w)\left(\left|\phi_{1}\right|^{2} \xi_{1}+\left|\phi_{2}\right|^{2} \xi_{2}+\cdots+\left|\phi_{n+1}\right|^{2} \xi_{n+1}+\cdots\right),
\end{aligned}
$$

where

$$
\xi_{n+1}=\frac{\sum_{r=0}^{n} \beta_{r}^{2}\left|\bar{p}_{r}(w)\right|}{\beta_{n+1}^{2}} \quad \forall n \in \mathbb{N} .
$$

Since $w \in\left(\operatorname{Int}\left(\mathscr{E}_{T}\right)\right)^{*}$, we have $\left|z_{1}(w)\right|<1$ and $\left|z_{2}(w)\right|<1$. Using the hypothesis that $\beta_{n}=\beta^{\sqrt{n}}$ with $\beta>1$, the sequence $\left\{\xi_{n}\right\}$ is bounded by the constant $P(w)$. Thus, the above computations show that $\psi(\lambda)=\phi(\lambda) /(\lambda-w)$ belongs to $H^{2}(G)$ whenever $\phi$ belongs to $H^{2}(G)$ with $\phi(w)=0$, and $\|\psi\|_{H^{2}}^{2} \leq P(w)^{2}\|\phi\|_{H^{2}}^{2}$. Next, we show that $\psi$ belongs to $H^{\infty}(G)$. Since $\phi$ belongs to $H^{\infty}(G)$, we have that $\phi k$ is in $H^{2}(G)$ with $(\phi k)(w)=0$, and therefore

$$
\|\psi k\|_{H^{2}}^{2} \leq P(w)^{2}\|\phi k\|_{H^{2}}^{2} \leq\|\phi\|_{\infty}^{2} P(w)^{2}\|k\|_{H^{2}}^{2} \quad \forall k \in H^{2}(G) .
$$

This proves that $\psi$ is in $H^{\infty}(G)$.

Lemma 3.3. If $\beta_{n}=\beta^{\sqrt{n}}$ with $\beta>1$, then $H^{\infty}(G)=H^{2}(G)$ and the norms $\|\cdot\|_{H^{2}},\|\cdot\|_{\infty}$ are equivalent.

Proof. The inclusion $H^{\infty}(G) \subseteq H^{2}(G)$ and the inequality $\|\phi\|_{H^{2}} \leq\|\phi\|_{\infty}$ were immediate consequences of the definition of $H^{\infty}(G)$. For the reverse inclusion, it is sufficient to show that $\|f g\|_{H^{2}} \leq C\|f\|_{H^{2}} \cdot\|g\|_{H^{2}}$, for some constant $C$ which is independent of $f, g \in H^{2}(G)$. Indeed, let $f(\lambda)=\sum_{n=0}^{\infty}\left(f_{n} / \beta_{n}\right) \bar{p}_{n}(\lambda)$ and $g(\lambda)=\sum_{n=0}^{\infty}\left(g_{n} / \beta_{n}\right) \bar{p}_{n}(\lambda)$ with $\sum_{n=0}^{\infty}\left|f_{n}\right|^{2}$ and $\sum_{n=0}^{\infty}\left|g_{n}\right|^{2}$ finite. Using an induction argument, one can deduce from formula (1.9) the following:

$$
\bar{p}_{r}(\lambda) \bar{p}_{s}(\lambda)=\bar{p}_{r+s}(\lambda)+b \bar{p}_{r-1}(\lambda) \bar{p}_{s-1}(\lambda) \quad \forall r, s \in \mathbb{N}^{*},
$$

which implies that

$$
\bar{p}_{s}(\lambda) \bar{p}_{n-s}(\lambda)=\bar{p}_{n}(\lambda)+b \bar{p}_{n-2}(\lambda)+\cdots+b^{s} \bar{p}_{n-2 s}(\lambda) \quad \forall n, s \in \mathbb{N}, 2 s \leq n .
$$


Applying formula (3.11) and an induction argument, one can deduce that $(f g)(\lambda)=$ $\sum_{s=0}^{\infty}(f g)_{s} \bar{p}_{s}(\lambda)$, where $(f g)_{s}=\sum_{n=0}^{\infty}(f g)_{s}^{n} b^{n}$ with

$$
(f g)_{s}^{n}=\sum_{t=0}^{s} \frac{f_{n+t}}{\beta_{n+t}} \cdot \frac{g_{n+s-t}}{\beta_{n+s-t}}
$$

Applying Cauchy's inequality we obtain

$$
\left|(f g)_{s}^{n}\right|^{2} \leq\left(\sum_{t=0}^{s}\left|f_{n+t} g_{n+s-t}\right|^{2}\right) \cdot\left(\sum_{t=0}^{s} \frac{1}{\beta_{n+t}^{2} \beta_{n+s-t}^{2}}\right) .
$$

Once again, by Cauchy's inequality we deduce

$$
\left|(f g)_{s}\right|^{2} \leq\left(\sum_{n=0}^{\infty}\left|(f g)_{s}^{n}\right|^{2} r^{n}\right) \cdot\left(\sum_{n=0}^{\infty} r^{n}\right)=\frac{1}{1-r}\left(\sum_{n=0}^{\infty}\left|(f g)_{s}^{n}\right|^{2} r^{n}\right),
$$

where $r=|b|$. Using the hypothesis that $\beta_{n}=\beta^{\sqrt{n}}$ with $\beta>1$, we have that (see [9, page 103])

$$
C:=\sup _{n \in \mathbb{N}}\left(\sum_{k=0}^{n} \frac{\beta_{n}^{2}}{\beta_{k}^{2} \beta_{n-k}^{2}}\right)<\infty .
$$

Therefore,

$$
\begin{aligned}
\|f g\|_{H^{2}}^{2} & =\sum_{s=0}^{\infty}\left|(f g)_{s}\right|^{2} \beta_{s}^{2} \\
& \leq \frac{1}{1-r} \sum_{s=0}^{\infty}\left(\sum_{n=0}^{\infty}\left[\sum_{t=0}^{s}\left|f_{n+t} g_{n+s-t}\right|^{2}\right] \cdot\left[\sum_{t=0}^{s} \frac{1}{\beta_{n+t}^{2} \beta_{n+s-t}^{2}}\right] \cdot r^{n}\right) \beta_{s}^{2} \\
& =\frac{1}{1-r} \sum_{s=0}^{\infty}\left(\sum_{n=0}^{\infty}\left[\sum_{t=0}^{s}\left|f_{n+t} g_{n+s-t}\right|^{2}\right] \cdot\left[\sum_{t=0}^{s} \frac{\beta_{s+2 n}^{2}}{\beta_{n+t}^{2} \beta_{n+s-t}^{2}}\right] \cdot r^{n} \frac{\beta_{s}^{2}}{\beta_{s+2 n}^{2}}\right) \\
& \leq \frac{C}{1-r} \sum_{s=0}^{\infty} \sum_{n=0}^{\infty} \sum_{t=0}^{s}\left|f_{n+t} g_{n+s-t}\right|^{2} \cdot r^{n} \frac{\beta_{s}^{2}}{\beta_{s+2 n}^{2}} \\
& =\frac{C}{1-r} \sum_{n=0}^{\infty} r^{n} \sum_{s=0}^{\infty} \frac{\beta_{s}^{2}}{\beta_{s+2 n}^{2}} \sum_{t=0}^{s}\left|f_{n+t} g_{n+s-t}\right|^{2} \\
& \leq \frac{C}{1-r} \sum_{n=0}^{\infty} r^{n}\left\|f-P_{n-1} f\right\|_{H^{2}}^{2} \cdot\left\|g-P_{n-1} g\right\|_{H^{2}}^{2} \\
& \leq \frac{C}{1-r} \sum_{n=0}^{\infty} r^{n}\|f\|_{H^{2}}^{2} \cdot\|g\|_{H^{2}}^{2} \\
& =\frac{C}{(1-r)^{2}}\|f\|_{H^{2}}^{2} \cdot\|g\|_{H^{2}}^{2},
\end{aligned}
$$

where $P_{n-1}$ is the orthogonal projection onto the subspace $\operatorname{span}\left\{\bar{f}_{i}(\lambda) \mid i=0, \ldots, n-1\right\}$. Thus $H^{2}(G) \subseteq H^{\infty}(G)$ and $\|k\|_{\infty} \leq\left(C /\left(1-r^{2}\right)\right)\|k\|_{H^{2}}$, for $k \in H^{2}(G)$. 
Lemma 3.4. If $\beta_{n}=\beta^{\sqrt{n}}, \beta>1$, then the maximal ideal space of $H^{\infty}(G)$ is $G$, and thus the spectrum of each element $\phi$ in $H^{\infty}(G)$ is $\phi(G)$.

Proof. According to Lemma 2.4, each $\lambda \in G$ is a bounded point evaluation, that is, the mapping $\phi \mapsto \phi(\lambda)$ from $H^{2}(G)$ to $\mathbb{C}$ is a continuous functional in the $H^{2}(G)$-norm, and according to Lemma 3.3, the functional is continuous in the $H^{\infty}(G)$-norm. Conversely, let $w=\Lambda\left(p_{1}\right)$, where $p_{1}(\lambda)=\lambda$ and $\Lambda$ is a character of $H^{\infty}(G)$. Thus $\Lambda(p)=$ $p(w)$, for any polynomial $p(\lambda)$. If $\phi(\lambda)=\sum_{n=0}^{\infty}\left(\phi_{n} / \beta_{n}\right) \bar{p}_{n}(\lambda)$ is an arbitrary element of $H^{\infty}(G)$, let $\phi^{n}(\lambda)=\sum_{j=0}^{n}\left(\phi_{j} / \beta_{j}\right) \bar{p}_{j}(\lambda)$. Thus, $\phi^{n} \rightarrow \phi$ in the $H^{2}(G)$ norm, and according to Lemma 3.3, $\phi^{n} \rightarrow \phi$ in the $H^{\infty}(G)$ norm. Therefore, $\Lambda\left(\phi^{n}\right) \rightarrow \Lambda(\phi)$. On the other hand $\Lambda\left(\phi^{n}\right)=\phi^{n}(w)=\left\langle\phi^{n}, k_{\bar{w}}\right\rangle \rightarrow\left\langle\phi, k_{\bar{w}}\right\rangle=\phi(w)$. Thus $\Lambda(\phi)=\phi(w)$. Furthermore, the series $\sum_{n=0}^{\infty}\left(\phi_{n} / \beta_{n}\right) \bar{p}_{n}(w)$ converges to a complex number $(\phi(w))$ if and only if $w \in G$.

When $H^{\infty}(G)=H^{2}(G)$, the function 1 is a strictly cyclic vector for the algebra $H^{\infty}(G)$ and therefore $H^{\infty}(G)$ is a maximal abelian algebra in $\mathscr{L}\left(H^{2}(G)\right)$. Thus, (cf. [9, page 92]), an element $\phi \in H^{\infty}(G)$ is invertible in $H^{\infty}(G)$ if and only if $M_{\phi}$ is invertible in $\mathscr{L}\left(H^{2}(G)\right)$. In combination with Lemma 3.4, we have the following.

Corollary 3.5. If $\phi \in H^{\infty}(G)$ and $\phi(\lambda) \neq 0$ for all $\lambda \in G$, then $M_{1 / \phi} \in \mathscr{L}\left(H^{2}(G)\right)$.

For a nonconstant function $\phi \in H^{\infty}(G)$, let $E_{\phi}$ be the set

$$
\left\{\lambda \in\left(\operatorname{Int}\left(\mathscr{E}_{T}\right)\right)^{*} \mid\left[\phi(\lambda) \notin \phi\left(\left(\mathscr{E}_{T}\right)^{*}\right)\right] \text { and }\left[\phi^{\prime}(w) \neq 0 \text { when } \phi(w)=\phi(\lambda)\right]\right\}
$$

The set $E_{\phi}$ is uncountable. To see this, observe first that the set

$$
\Omega_{1}:=\left\{\lambda \in\left(\operatorname{Int}\left(\mathscr{E}_{T}\right)\right)^{*} \mid \phi(\lambda) \notin \phi\left(\left(\mathscr{E}_{T}\right)^{*}\right)\right\}
$$

is a nonempty open set. On the other hand, if one denotes

$$
\Omega_{2}:=\left\{\lambda \in\left(\operatorname{Int}\left(\mathscr{E}_{T}\right)\right)^{*} \mid \phi^{\prime}(w)=0 \text { when } \phi(w)=\phi(\lambda)\right\}
$$

then $\Omega_{1}^{n} \cap \Omega_{2}$ is a finite set, where $\cup_{n \in \mathbb{N}} \Omega_{1}^{n}$ is an open increasing exhaustion (with $\overline{\Omega_{1}^{n}} \subset$ $\Omega_{1}$ ) of $\Omega_{1}$. Thus, $\Omega_{1} \cap \Omega_{2}$ is a countable set and therefore $\Omega_{1} \cap \Omega_{2}^{c}$, that is, $E_{\phi}$ is uncountable and thus nonempty.

We need the following statement which we leave as an open problem.

Conjecture 3.6. If $\phi \in H^{\infty}(G)$ has infinitely many different zeros in $G$, then $\phi=0$.

The above statement was obtained by Carleson [3] for functions in $H^{2}(\beta)$. We only point here that there are functions in the disc algebra which vanish on a Cantor set of the unit circle and which are not entirely zero. In spite of the fact that Conjecture 3.6 has 
an elementary statement, its proof seems not to be elementary and the circle of ideas used in [3] are not applicable in this case.

For $\phi \in H^{\infty}(G)$ and $\lambda \in E_{\phi}$, let $\mathcal{M}_{\lambda, \phi}$ be the nullspace of $[\phi(T)-\phi(\lambda)]^{*}$. The following lemma is the equivalent of [6, Lemma 2$]$ and this is the first place in which Conjecture 3.6 is used.

Lemma 3.7. If $\phi \in H^{\infty}(G)$ is a nonconstant function and $\lambda \in E_{\phi}$, then $M_{\lambda, \phi}=\operatorname{span}\left\{f_{\bar{w}} \mid\right.$ $w \in\left(\operatorname{Int}\left(\mathscr{E}_{T}\right)\right)^{*}$ such that $\left.\phi(w)=\phi(\lambda)\right\}$.

Proof. For an arbitrary $h \in l^{2}(\mathbb{N})$ we have

$$
\begin{aligned}
\left\langle h,[\phi(T)-\phi(\lambda)]^{*} f_{\bar{w}}\right\rangle_{l^{2}(\mathbb{N})} & =\left\langle[\phi(T)-\phi(\lambda)] h, f_{\bar{w}}\right\rangle_{l^{2}(\mathbb{N})}=(U[\phi(T)-\phi(\lambda)] h)(w) \\
& =\left(M_{\phi}-\phi(\lambda)\right) U h(w)=(\phi(w)-\phi(\lambda)) U h(\cdot) .
\end{aligned}
$$

Thus, for $\phi \in H^{\infty}(G), \lambda \in E_{\phi}$, and $w \in G$ such that $\phi(w)=\phi(\lambda)$, we have

$$
\left\langle h,[\phi(T)-\phi(\lambda)]^{*} f_{\bar{w}}\right\rangle_{l^{2}(\mathbb{N})}=0 .
$$

This establishes that

$$
M_{\lambda, \phi} \supseteqq \vee\left\{f_{\bar{w}} \mid w \in\left(\operatorname{Int}\left(\mathscr{E}_{T}\right)\right)^{*} \text { such that } \phi(w)=\phi(\lambda)\right\} \text {. }
$$

For the inverse inclusion it is enough to show

$$
\begin{aligned}
& {\left[h \in l^{2}(\mathbb{N}) \text { such that } U h(w)=0 \text { for } w \in\left(\operatorname{Int}\left(\mathscr{E}_{T}\right)\right)^{*} \text { with } \phi(w)=\phi(\lambda)\right.} \\
& \quad \Longrightarrow h \in \operatorname{Ran}(\phi(T)-\phi(\lambda))]
\end{aligned}
$$

or equivalently,

$$
\begin{aligned}
{[k} & \in H^{2}(G) \text { such that } k(w)=0 \text { for } w \in\left(\operatorname{Int}\left(\mathscr{E}_{T}\right)\right)^{*} \text { with } \phi(w)=\phi(\lambda) \\
& \left.\Longrightarrow \text { there exists some } k^{\prime} \text { in } H^{2}(G) \text { such that } k=(\phi-\phi(\lambda)) k^{\prime}\right] .
\end{aligned}
$$

Let $\lambda \in E_{\phi}$ and $k \in H^{2}(G)$ be such that $k(w)=0$ for $w \in\left(\operatorname{Int}\left(\mathscr{E}_{T}\right)\right)^{*}$ with $\phi(w)=\phi(\lambda)$. Since according to Conjecture 3.6, the set $\{w \mid \phi(w)=\phi(\lambda)\}$ is finite, we can define $p(z)=$ $\Pi\{(z-w) \mid \phi(w)=\phi(\lambda)\}$. Applying Lemma 3.2, we can write $k=p k_{1}$ with $k_{1} \in H^{2}(G)$ and $\phi-\phi(\lambda)=p \phi_{1}$ with $\phi_{1} \in H^{\infty}(G)$. Since $\phi-\phi(\lambda)$ has only simple zeros, $\phi_{1}$ does not vanish on $G$, and thus, according to Corollary $3.5,1 / \phi_{1}$ belongs to $H^{\infty}(G)$. Therefore $k^{\prime}:=k_{1} / \phi_{1}$ belongs to $H^{2}(G)$ and $\left[M_{\phi}-\phi(\lambda)\right] k^{\prime}=k$.

The proof of the main theorem of [6] can be repeated for the class of operators discussed in this paper, and for the sake of completeness, we include it here.

First, the following lemma (whose proof is omitted) about Schur product is needed. The Schur product of two matrices $A=\left(a_{i j}\right)$ and $B=\left(b_{i j}\right)$ denoted by $A \cdot B$ is the matrix $\left(a_{i j} b_{i j}\right)$. 
Lemma 3.8 [6]. If $A$ is an $n \times n$ matrix such that $A \cdot B$ is a nilpotent matrix for any $n \times n$ matrix $B$, then at least one of the columns of $A$ has all entries equal to 0.

The proof of Theorem 3.9 makes use of Conjecture 3.6 and Lemma 3.7, and implicitly, the content of subsequent statements, Theorem 3.10 and Corollary 3.11, are dependent on the same conjecture.

Theorem 3.9 [6]. Let $\phi(T)$ be an operator in the commutant of $T$ such that $\phi$ is a nonconstant function of $H^{\infty}(G)$. Then the only compact operator that commutes with $\phi(T)$ is equal to zero.

Proof. First, it is shown that there exists an integer $n_{0}$ such that $K^{n_{0}}=0$ for any compact operator $K$ commuting with $\phi(T)$. Indeed, for a compact operator $K$ that commutes with $\phi(T)$ with $\phi$ as in the hypothesis of theorem, $K^{*}$ commutes with $\phi(T)^{*}$, and thus by Lemma 3.7, the subspace $M_{\lambda, \phi}$ is invariant for $K^{*}$. Since the set $E_{\phi}$ is uncountable and the subspace $M_{\lambda, \phi}$ is finite-dimensional for $\lambda \in E_{\phi}$, there exists an integer $n_{0}$ such that the dimension of $M_{\lambda, \phi}$ is $n_{0}$ for uncountably many $\lambda^{\prime}$ s in $E_{\phi}$. The restriction operator $K^{*} \mid M_{\lambda, \phi}$ has an eigenvalue. For $\lambda, \lambda^{\prime}$ such that $\phi(\lambda) \neq \phi\left(\lambda^{\prime}\right)$, by Lemma 3.7, $M_{\lambda, \phi} \cap M_{\lambda^{\prime}, \phi}=\{0\}$. Recalling the spectral properties of a compact operator and using the above observations, one can conclude that $\sigma\left(K^{*} \mid M_{\lambda, \phi}\right)=\{0\}$ for uncountably many $\lambda$ 's for which the dimension of $M_{\lambda, \phi}$ is equal to $n_{0}$. Thus, for such $\lambda^{\prime}$ 's, we have $\left(K^{*} \mid M_{\lambda, \phi}\right)^{n_{0}}=0$; in particular, $\left(K^{*}\right)^{n_{0}} f_{\bar{\lambda}}=0$ for infinitely many $\lambda$ 's. Since the span of infinitely many $f_{\bar{\lambda}}$ 's corresponding to different $\lambda$ 's is the entire $H^{2}(G)$ (easy consequence of Lemma 2.4 and Conjecture 3.6), one concludes that $\left(K^{*}\right)^{n_{0}}=0$, and thus $K^{n_{0}}=0$.

Now, one can prove the actual statement of the theorem. Let $K_{0}$ be a compact operator in the commutant of $\phi(T)$. For any polynomials $p$ and $q$, the operator $p(T)^{*} K_{0}^{*} q(T)^{*}$ commutes with $\phi(T)^{*}$ and is compact. Let $\lambda \in E_{\phi}$ and $\left\{f_{\bar{w}_{1}}, \ldots, f_{\bar{w}_{n_{0}}}\right\}$ be a basis of $\mathcal{M}_{\lambda, \phi}$. Since $T^{*} f_{\bar{w}}=\bar{w} f_{\bar{w}}$, the restriction $T^{*} \mid M_{\lambda, \phi}$ has diagonal matrix representation with respect to the above basis. One can choose the polynomials $p_{i}$, for each $i \in\left\{1, \ldots, n_{0}\right\}$ such that $p_{i}\left(T^{*}\right) \mid \mathcal{M}_{\lambda, \phi}$ is the orthogonal projection $P_{i}$ onto $\mathbb{C} f_{\bar{w}_{i}}$ along $\operatorname{span}\left\{f_{\bar{w}_{j}} \mid j \neq i\right\}$. Thus, for any $n_{0} \times n_{0}$ matrix $\left(b_{i j}\right)$, the operator $\sum_{i, j=1}^{n_{0}} b_{i j} P_{i} K_{0} P_{j}$ on $M_{\lambda, \phi}$ is the restriction to $M_{\lambda, \phi}$ of $\sum_{i, j=1}^{n_{0}} b_{i j} p_{i}\left(T^{*}\right) K_{0} p_{j}\left(T^{*}\right)$, which, by the first part of this proof, must be a nilpotent operator of order $n_{0}$. Since this operator is the Schur product of the matrices $\left(b_{i j}\right)$ and $K_{0}^{*} \mid M_{\lambda, \phi}$, according to Lemma 3.8, at least one of the columns of $K_{0}^{*} \mid M_{\lambda, \phi}$ with respect to $\left\{f_{\bar{w}_{1}}, \ldots, f_{\bar{w}_{n_{0}}}\right\}$ is zero, that is, there exists $f_{\bar{w}_{j}} \in M_{\lambda, \phi}$ such that $K_{0}^{*} f_{\bar{w}_{j}}=0$. Thus $K_{0}^{*} f_{\bar{w}}=0$ for infinitely many $w$ 's, and therefore $K_{0}^{*}=0$ and $K_{0}=0$.

Theorem 3.10. The class of weighted Toeplitz operators of symbol in $\varphi$ is included in $\tilde{S} \backslash S$.

The fact that $\phi(T)$ belongs to $\widetilde{S}$ is a consequence of main theorem of [8] and that $\phi(T)$ does not belong to set $S$ results from Theorem 3.9.

Corollary 3.11. Each weighted Toeplitz operator of symbol $\psi(z)=a z^{k}+\left(b / z^{k}\right), k \in \mathbb{N}^{*}$, $|a|>|b|$, belongs to $\tilde{S} \backslash S$.

Proof. If $k>1$ and $\psi(z)=a z^{k}+b / z^{k}$, then $T_{\psi}^{(\beta)}$ is unitarily equivalent to

$$
T_{\phi}^{\left(\beta^{0}\right)} \oplus T_{\phi}^{\left(\beta^{1}\right)} \oplus \cdots \oplus T_{\phi}^{\left(\beta^{k-1}\right)}
$$


where $\beta^{0}=\left(\beta_{n k}\right)_{n \in \mathbb{N}}, \beta^{1}=\left(\beta_{1+n k}\right)_{n \in \mathbb{N}}, \ldots, \beta^{k-1}=\left(\beta_{k-1+n k}\right)_{n \in \mathbb{N}}$, and $\phi(z)=a z+b / z$. Thus, Corollary 3.11 is a straightforward consequence of Theorem 3.10.

The shift operator provided in [6] $\alpha$-commutes with a nonzero compact operator, and consequently, the set of operators that satisfy the hypothesis of Theorem 3.1 is significantly larger than the set of operators that satisfy Lomonosov's result.

\section{References}

[1] A. Biswas, A. Lambert, and S. Petrovic, Extended eigenvalues and the Volterra operator, Glasg. Math. J. 44 (2002), no. 3, 521-534.

[2] S. Brown, Connections between an operator and a compact operator that yield hyperinvariant subspaces, J. Operator Theory 1 (1979), no. 1, 117-121.

[3] L. Carleson, Sets of uniqueness for functions regular in the unit circle, Acta Math. 87 (1952), 325-345.

[4] J. B. Conway and G. Prǎjiturǎ, On ג-commuting operators, Studia Math. 166 (2005), no. 1, 1-9.

[5] P. L. Duren, Extension of a result of Beurling on invariant subspaces, Trans. Amer. Math. Soc. 99 (1961), 320-324.

[6] D. W. Hadwin, E. A. Nordgren, H. Radjavi, and P. Rosenthal, An operator not satisfying Lomonosov's hypothesis, J. Funct. Anal. 38 (1980), no. 3, 410-415.

[7] H. W. Kim, R. Moore, and C. M. Pearcy, A variation of Lomonosov's theorem, J. Operator Theory 2 (1979), no. 1, 131-140.

[8] V. Lauric, Operators $\alpha$-commuting with a compact operator, Proc. Amer. Math. Soc. 125 (1997), no. 8, 2379-2384.

[9] A. L. Shields, Weighted shift operators and analytic function theory, Topics in Operator Theory, Math. Surveys, no. 13, American Mathematical Society, Rhode Island, 1974, pp. 49-128.

Vasile Lauric: Department of Mathematics, Florida A\&M University, Tallahassee, FL 32307, USA E-mail address: vasile.lauric@famu.edu 


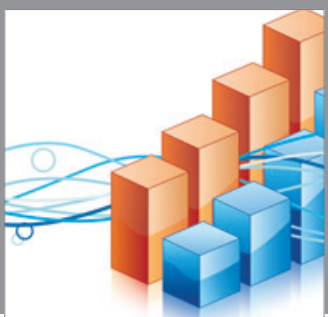

Advances in

Operations Research

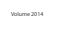

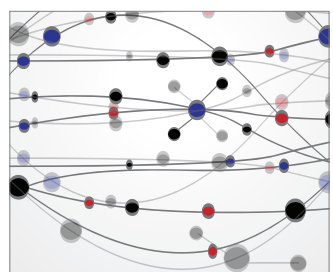

\section{The Scientific} World Journal
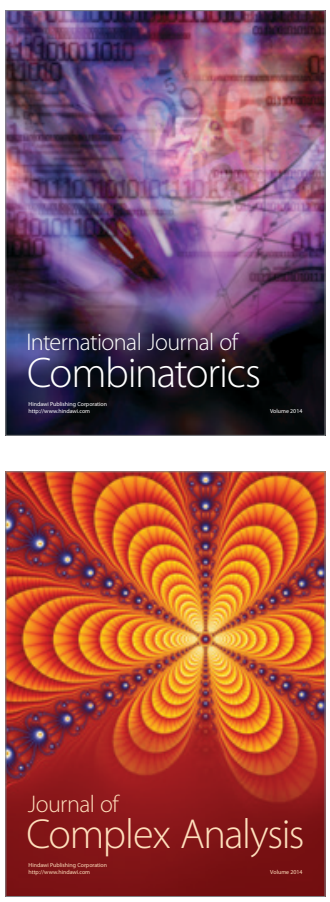

International Journal of

Mathematics and

Mathematical

Sciences
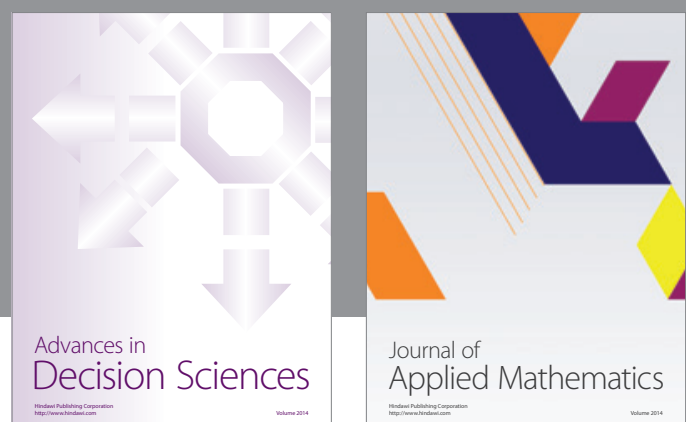

Journal of

Applied Mathematics
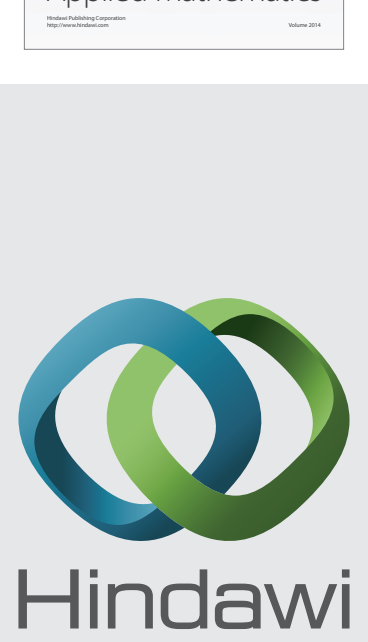

Submit your manuscripts at http://www.hindawi.com
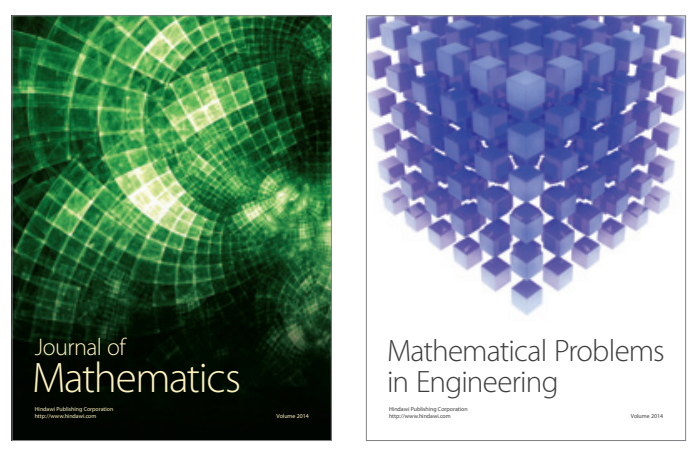

Mathematical Problems in Engineering
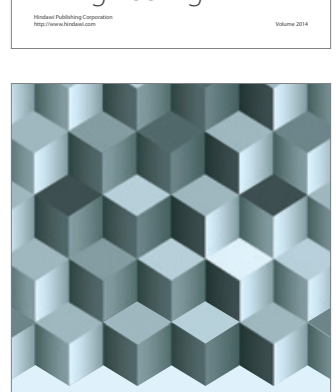

Journal of

Function Spaces
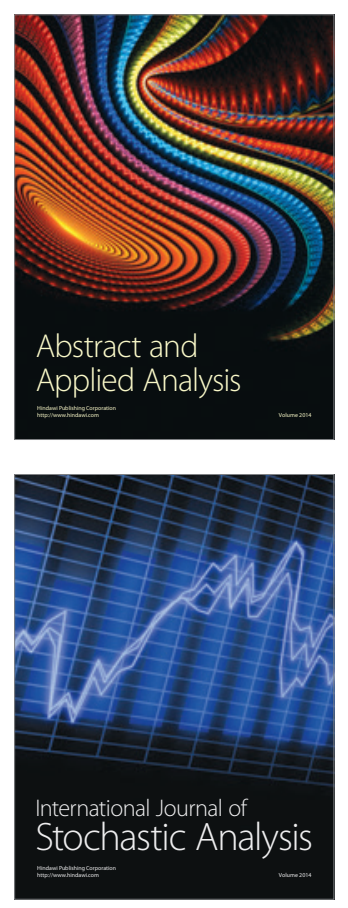

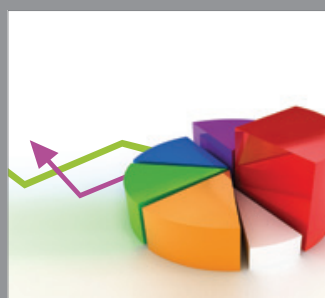

ournal of

Probability and Statistics

Promensencen
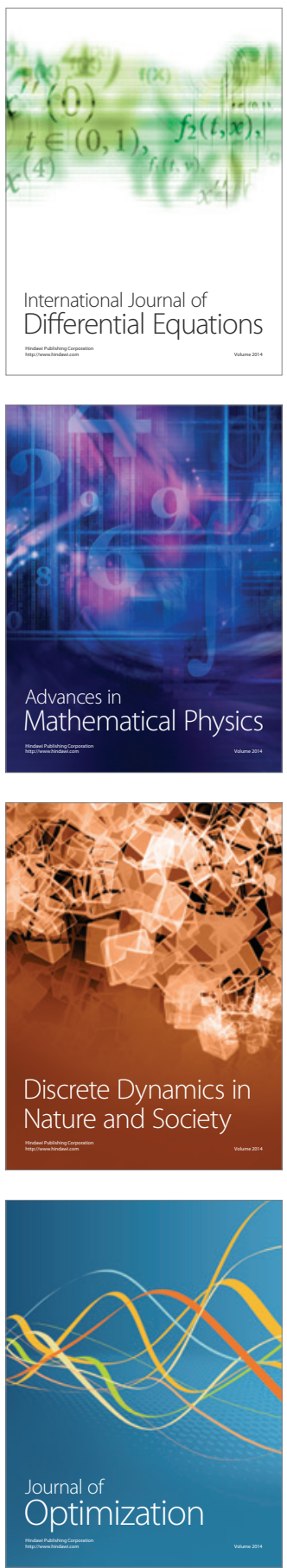